\title{
An integral formula for bi-slant submanifolds in complex space forms
}

\author{
Aliya Naaz Siddiqui ${ }^{1}$ (D), Cihan Özgür*2 (D) \\ ${ }^{1}$ Department of Mathematics, Faculty of Natural Sciences, Jamia Millia Islamia, New Delhi - 110025, \\ India \\ ${ }^{2}$ Department of Mathematics, Balıkesir University, 10145, Ballkesir, Turkey
}

\begin{abstract}
The present paper deals with bi-slant submanifolds in complex space forms. We obtain an integral formula of Simons' type for bi-slant submanifolds of a complex space form with positive constant holomorphic sectional curvature. Then, we apply it to prove our main result. We also discuss the same result in different submanifolds such as hemi-slant and semi-slant submanifolds.
\end{abstract}

Mathematics Subject Classification (2010). 53C15, 53C25, 53C40, 53C 42

Keywords. bi-slant submanifolds, minimal submanifolds, totally geodesic submanifolds, complex space forms

\section{Introduction}

As a generalization of totally real submanifolds and holomorphic submanifolds, $C R$ submanifolds of Kaehler manifolds were introduced by Bejancu in [2]. Since then, such submanifolds have attracted many geometers (see for example $[3,8,11,16,22]$ ). As another interesting subject, the notion of a slant submanifold in an almost Hermitian manifold was introduced by B.-Y. Chen in $[9,10]$. A. Lotta introduced the notion of slant immersions of Riemannian manifolds into an almost contact metric manifolds. Afterwards, these submanifolds were studied by J.L. Cabrerizo et al. in the setting of Sasakian manifolds. Now, it has become a very active research area in both complex geometry and contact geometry (see for example [5, 6, 20]). Further, N. Papaghiuc [17] defined semi-slant submanifolds as a generalization of slant and $C R$-submanifolds. Slant and semi-slant submanifolds are a generalization of invariant, anti-invariant, semi-invariant and totally real submanifolds. In [4], J.L. Cabrerizo et al. generalized these notions by introducing bi-slant submanifolds in almost Hermitian manifolds. One of the classes of bi-slant submanifolds is that of anti-slant submanifolds which are studied by J.L. Cabrerizo et al. B. Şahin [20] named these submanifolds as hemi-slant submanifolds and also called pseudo-slant submanifolds. Since then, many geometers have studied these types of submanifolds in different ambient spaces.

J. Simons [19] studied the basic exposition of immersed minimal varieties in a Riemannian manifold. The principal result of this general investigation became the derivation of

\footnotetext{
*Corresponding Author.

Email addresses: aliyanaazsiddiqui9@gmail.com (A.N. Siddiqui), cozgur@balikesir.edu.tr (C. Özgür)

Received: 25.07.2019; Accepted: 23.03.2020
} 
the linear elliptic second order equation, which was satisfied by the second fundamental form of any minimal variety in any ambient manifold. In 1981, A. Bejancu et al. [3] derived Simons' type formula for $C R$-submanifolds of a complex space form. Since then, many authors derived Simons' type formula for different submanifolds in ambient spaces (see for example [14,15,21]). Recently, A.N. Siddiqui et al. [18] studied Simons' type formula for Kaehlerian slant submanifolds in complex space forms. The purpose of the present paper is to continue the study of bi-slant submanifolds and to construct Simons' type formula for bi-slant, hemi-slant, and semi-slant submanifolds of complex space forms.

The paper has been organized as follows: Section 2 is preliminary in nature. Some examples of semi-slant, hemi-slant, and bi-slant submanifolds in complex space forms are also discussed. In Section 3, some lemmas are quoted for later use. We derive Simons' type formula for a minimal bi-slant submanifold in a complex space form with positive constant holomorphic sectional curvature and also construct for hemi-slant and semi-slant submanifolds in the same ambient space. In Section 4, we prove the main result by using an integral formula of Simons' type and Hopf's Lemma. Some immediate consequences are also obtained.

\section{Preliminaries}

Let $M$ be an $n$-dimensional submanifold of a Riemannian manifold $(\bar{M}, g)$ and $\nabla, \bar{\nabla}$ denote the induced Riemannian and Riemannian connections on $M$ and $\bar{M}$, respectively. The Gauss and Weingarten formulas are given by $\bar{\nabla}_{X} Y=\nabla_{X} Y+B(X, Y)$ and $\bar{\nabla}_{X} V=$ $-A_{V}(X)+\nabla \frac{1}{X} V$, for any vector fields $X, Y$ tangent to $M$ and a vector field $V$ normal to $M$, where $\nabla^{\perp}$ denotes the normal connection, $B$ the second fundamental form and $A_{V}$ the shape operator in the direction of $V$. If $B=0$, then $M$ is called totally geodesic [8]. Let $p \in M$ and $\left\{e_{1}, \ldots, e_{n}\right\}$ be an orthonormal basis of $T_{p} M$ and $\left\{e_{n+1}, \ldots, e_{2 n}\right\}$ an orthonormal basis of $T_{p}^{\perp} M$. The mean curvature vector $H$ of a submanifold $M$ at $p$ is defined by

$$
H(p)=\frac{1}{n} \sum_{i=1}^{n} B\left(e_{i}, e_{i}\right)
$$

If $H=0$, i.e., trace $B \equiv 0$, then $M$ is called minimal [8].

Denote by $\bar{R}$ and $R$ the curvature tensor fields of $\bar{M}$ and $M$, respectively. Then the Gauss equation is given by

$$
\bar{R}(X, Y, Z, W)=R(X, Y, Z, W)-g\left(A_{B(Y, Z)} X, W\right)+g\left(A_{B(X, Z)} Y, W\right),
$$

for all vector fields $X, Y, Z, W$ tangent to $M$ [8].

For the Laplacian of the second fundamental form $A$ of an $n$-dimensional minimal submanifold $M$ in an $m$-dimensional Riemannian manifold $\bar{M}$, the following Simons' type formula is well known ([19], pg - 81):

$$
\nabla^{2} A=-A \circ \tilde{A}-\underset{\sim}{A} \circ A+\bar{R}(A)+\overline{R^{\prime}}
$$

where the operators $\tilde{A}$ and $\underset{\sim}{A}$ are defined as follows:

$$
\tilde{A}=A^{t} \circ A \text { and } \underset{\sim}{A}=\sum_{a=1}^{m-n} a d A_{a} a d A_{a}
$$

for a normal frame $\left\{e_{a}\right\}, a=1, \ldots, m-n$, and $A_{a}=A_{e_{a}}$, where $\bar{R}(A)$ and $\overline{R^{\prime}}$ are given by the following relations:

$$
\begin{gathered}
g\left(\bar{R}(A)^{N}(X), Y\right)=\sum_{i=1}^{n}\left[2 g\left(\bar{R}\left(e_{i}, Y\right) B\left(X, e_{i}\right), N\right)+2 g\left(\bar{R}\left(e_{i}, X\right) B\left(Y, e_{i}\right), N\right)\right. \\
-g\left(A_{N}(X), \bar{R}\left(e_{i}, Y\right) e_{i}\right)-g\left(A_{N}(Y), \bar{R}\left(e_{i}, X\right) e_{i}\right)
\end{gathered}
$$




$$
\left.+g\left(\bar{R}\left(e_{i}, B(X, Y)\right) e_{i}, N\right)-2 g\left(A_{N}\left(e_{i}\right), \bar{R}\left(e_{i}, X\right) Y\right)\right]
$$

and

$$
g\left({\overline{R^{\prime}}}^{N}(X), Y\right)=\sum_{i=1}^{n}\left(g\left(\left(\bar{\nabla}_{X} \bar{R}\right)\left(e_{i}, Y\right) e_{i}, N\right)+g\left(\left(\bar{\nabla}_{e_{i}} \bar{R}\right)\left(e_{i}, X\right) Y, N\right)\right)
$$

for all vector fields $X, Y$ tangent to $M$ and $N$ normal to $M$.

An almost Hermitian manifold $\bar{M}$ with an almost complex structure $J$ is called a Kaehler manifold if $J$ is parallel with respect to the Levi-Civita connection $\bar{\nabla}$ of $g$, i.e., $\bar{\nabla} J=0$.

A Kaehler manifold of constant holomorphic sectional curvature $c$ is called a complex space form and it is denoted by $\bar{M}(c)$ [23]. The Riemannian curvature tensor field of $\bar{M}(c)$ is given by

$$
\begin{aligned}
\bar{R}(X, Y) Z= & \frac{c}{4}\{g(Y, Z) X-g(X, Z) Y+g(X, J Z) J Y \\
& -g(Y, J Z) J X+2 g(X, J Y) J Z\},
\end{aligned}
$$

for any vector fields $X, Y, Z$ tangent to $\bar{M}(c)$ [23].

Now, let $\bar{M}$ be a real $2 n$-dimensional Kaehler manifold with complex structure $J$ and $M$ be an isometrically immersed submanifold in $\bar{M}$. For any vector field $X$ tangent to $M$, we put

$$
J X=P X+F X,
$$

where $P X$ and $F X$ denote the tangential and normal components of $J X$, respectively. Then $P$ is an endomorphism of $T M$ and $F$ is the normal bundle valued 1-form on $T M$.

For any vector field $V$ normal to $M$, we put

$$
J V=t V+f V,
$$

where $t V$ and $f V$ denote tangential and normal components of $J V$, respectively.

By the use of Gauss equation (2.1) and (2.5), we can write the Riemannian curvature tensor field $R$ of $M$ as

$$
\begin{aligned}
R(X, Y, Z, W)= & \frac{c}{4}\{g(Y, Z) g(X, W)-g(X, Z) g(Y, W) \\
& +g(P Y, Z) g(P X, W)-g(P X, Z) g(P Y, W) \\
& +2 g(X, P Y) g(P Z, W)\}+g\left(A_{B(Y, Z)} X, W\right) \\
& -g\left(A_{B(X, Z)} Y, W\right)
\end{aligned}
$$

for any vector fields $X, Y, Z$ tangent to $T M$.

A submanifold $M$ of an almost Hermitian manifold $\bar{M}$ is called slant [9], if for each non-zero vector $X$ tangent to $M$, the angle $\theta(X)$ between $J X$ and $T_{p} M$ is a constant, i.e., it does not depend on the choice of $p \in M$ and $X \in T_{p} M$.

Furthermore,

(i) A submanifold $M$ of $\bar{M}$ is called a semi-slant submanifold [17] of $\bar{M}$, if there exists a pair of orthogonal distributions $D$ and $D_{\theta}$ such that $D$ is holomorphic and $D_{\theta}$ is proper slant.

(ii) A submanifold $M$ of $\bar{M}$ is called a hemi-slant submanifold (or pseudo-slant) [7] of $\bar{M}$, if there exists a pair of orthogonal distributions $D_{\perp}$ and $D_{\theta}$ such that $D_{\perp}$ is totally real and $D_{\theta}$ is proper slant.

(iii) A submanifold $M$ of $\bar{M}$ is called a bi-slant submanifold [4] of $\bar{M}$, if there exists a pair of orthogonal distributions $D_{\theta_{1}}$ and $D_{\theta_{2}}$ such that $D_{\theta_{1}}$ is proper slant with slant angle $\theta_{1}$ and $D_{\theta_{2}}$ is proper slant with slant angle $\theta_{2}$.

Now we give some examples of bi-slant, semi-slant and hemi-slant submanifolds of complex space forms. 
Example 2.1. [13] Let $\left(\mathbb{R}^{2 m}, J\right)$ be the Euclidean $2 m$-space and endowed with the Euclidean metric $g$, where $J$ is an almost complex structure on $\mathbb{R}^{2 m}$. The Euclidean metric $g$ is defined by

$$
\begin{aligned}
& g\left(\left(x_{1}, \ldots, x_{m}, y_{1}, \ldots, y_{m}\right),\left(z_{1}, \ldots, z_{m}, w_{1}, \ldots, w_{m}\right)\right) \\
= & x_{1} z_{1}+\cdots+x_{m} z_{m}+y_{1} w_{1}+\cdots+y_{m} w_{m} .
\end{aligned}
$$

Now let $\varphi: M \rightarrow \mathbb{R}^{6}$ defined by

$$
\varphi(u, v, w, t)=(u \sqrt{2}, v \sqrt{2}, u+v, u-v, w-t, t-w) .
$$

Then we have an orthonormal frame $\left\{e_{1}, e_{2}, e_{3}, e_{4}\right\}$ of $T_{p} M, p \in M$ such that

$$
\begin{aligned}
& e_{1}=\frac{1}{2}(\sqrt{2}, 0,1,1,0,0), \\
& e_{2}=\frac{1}{2}(0, \sqrt{2}, 1,-1,0,0), \\
& e_{3}=\frac{1}{\sqrt{2}}(0,0,0,0,1,-1), \\
& e_{4}=\frac{1}{\sqrt{2}}(0,0,0,0,0,-1,1) .
\end{aligned}
$$

Thus, $M$ is a totally geodesic bi-slant submanifold of $\mathbb{R}^{6}$ such that $D_{\theta_{1}}=\operatorname{span}\left\{e_{1}, e_{2}\right\}$ with slant angle $\alpha$ and $D_{\theta_{2}}=\operatorname{span}\left\{e_{3}, e_{4}\right\}$ with slant angle $\beta$, where

$$
J(a, b, c, d, e, f)=(b,-a, d,-c, f,-e) .
$$

Using [12] (see page 10), we can state the following four examples.

Example 2.2. Let $\bar{M}(c)$ be a complex space form with two orthogonal submanifolds $M_{\theta}$, a slant submanifold with slant angle $\theta \neq \frac{\pi}{2}$, and $M_{T}$ a holomorphic submanifold. Then the Riemannian product $M=M_{\theta} \times M_{T}$ is a semi-slant submanifold of $\bar{M}(c)$.

Example 2.3. Let $\bar{M}(c)$ be a complex space form with two orthogonal submanifolds $M_{\theta}$, a slant submanifold with slant angle $\theta \neq \frac{\pi}{2}$, and $M_{\perp}$ a totally real submanifold. Then the Riemannian product $M=M_{\perp} \times M_{\theta}$ is a hemi-slant submanifold of $\bar{M}(c)$.

Example 2.4. Let $\bar{M}(c)$ be a complex space form with two orthogonal submanifolds $M_{\theta}$, a slant submanifold with slant angle $\theta$, and $M_{T}$ a holomorphic submanifold. Then the Riemannian product $M=M_{\theta} \times M_{T}$ is a bi-slant submanifold of $\bar{M}(c)$.

Example 2.5. Let $\bar{M}(c)$ be a complex space form with two orthogonal submanifolds $M_{\theta_{1}}$ and $M_{\theta_{2}}$ such that $M_{\theta_{2}}$ and $M_{\theta_{2}}$ are slant submanifolds of $\bar{M}(c)$ with slant angles $\theta_{1}$ and $\theta_{2}$, respectively. Then the Riemannian product $M=M_{\theta_{1}} \times M_{\theta_{2}}$ is a bi-slant submanifold of $\bar{M}(c)$.

Remark 2.6. It is easy to see that if $M_{1}$ and $M_{2}$ are minimal submanifolds, then the Riemannian product $M=M_{1} \times M_{2}$ is also a minimal submanifold. Hence if we take $M_{1}$ and $M_{2}$ as minimal submanifolds in the above four examples, we will have minimal submanifolds.

\section{Simons' type formula}

This section is devoted to some preparatory results while our main result of this paper is given in the next section.

Lemma 3.1. [9] Let $M$ be any submanifold of a Kaehler manifold $\bar{M}$. Then $\bar{\nabla} P=0$ if and only if

$$
A_{F X}(Y)=A_{F Y}(X)
$$

for any vector fields $X, Y$ tangent to $M$. 
Lemma 3.2. [9] Let $M$ be any submanifold of a Kaehler manifold $\bar{M}$. Then $\bar{\nabla} F=0$ if and only if

$$
A_{f N}(X)=-A_{N}(P X),
$$

for any vector fields $X$ tangent to $M$ and $N$ normal to $M$.

Lemma 3.3. [8] Let $M$ be any submanifold of a Kaehler manifold $\bar{M}$. If $\bar{\nabla} P=0$, then

$$
A_{N} P+P A_{N}=0,
$$

for any vector field $N$ normal to $M$.

For a bi-slant submanifold $M$ of dimension $n=2 n_{1}+2 n_{2}$ in a complex space form $\bar{M}(c)$, we have

$$
T M=D_{\theta_{1}} \oplus D_{\theta_{2}}
$$

then

$$
\|P\|^{2}=\sum_{i, j=1}^{n} g^{2}\left(P e_{i}, e_{j}\right)=2\left(n_{1} \cos ^{2} \theta_{1}+n_{2} \cos ^{2} \theta_{2}\right),
$$

where $\operatorname{dim}\left(D_{\theta_{1}}\right)=2 n_{1}$ and $\operatorname{dim}\left(D_{\theta_{2}}\right)=2 n_{2}$.

Using above Lemmas and equations (2.2), (2.3) and (2.4), we obtain the following result to get the required Simons' type formula for an $n$-dimensional minimal bi-slant submanifold in a complex space form $\bar{M}(c)$ of dimension $2 n$, also for hemi-slant and semislant submanifolds, which play crucial role in working out the main results of this paper.

Theorem 3.4. Let $M$ be an $n$-dimensional minimal bi-slant submanifold of a complex space form $\bar{M}(c)$ of dimension $2 n$. If $\bar{\nabla} P=0$, then

$$
\begin{gathered}
g\left(\nabla^{2} A, A\right)=-g(A \circ \tilde{A}+\underset{\sim}{A} \circ A, A) \\
+\frac{c}{4}\left[n+1+6\left(n_{1} \cos ^{2} \theta_{1}+n_{2} \cos ^{2} \theta_{2}\right)\right]\|A\|^{2} .
\end{gathered}
$$

Proof. Since $\bar{M}(c)$ is locally symmetric, i.e., $\bar{\nabla} \bar{R}=0$, then (2.4) implies

$$
g\left({\overline{R^{\prime}}}^{N}(X), Y\right)=0 .
$$

Now, we compute $g\left(\bar{R}(A)^{N}(X), Y\right)$ by putting (2.5) into (2.3) and then from Lemmas 3.1-3.3, we get the following easily,

$$
\begin{gathered}
g\left(\bar{R}(A)^{N}(X), Y\right)=\frac{c}{4}\left[n g\left(A_{N}(X), Y\right)-2 g\left(A_{F Y}(X), t N\right)-2 g\left(A_{F X}(Y), t N\right)\right. \\
-4 g\left(A_{f N}(X), P Y\right)-4 g\left(A_{f N}(Y), P X\right)+3 g\left(P X, P A_{N}(Y)\right) \\
+3 g\left(P Y, P A_{N}(X)\right)-6 g\left(A_{N}(P X), P Y\right)-2 \sum_{i=1}^{n}\left(g\left(A_{F e_{i}}\left(e_{i}\right), X\right) g(F Y, N)\right. \\
\left.\left.+g\left(A_{F e_{i}}\left(e_{i}\right), Y\right) g(F X, N)+\frac{3}{2} g\left(A_{F e_{i}}(X), Y\right) g\left(F e_{i}, N\right)\right)\right] \\
=\frac{c}{4}\left[n-3+14\left(n_{1} \cos ^{2} \theta_{1}+n_{2} \cos ^{2} \theta_{2}\right)\right] g\left(A_{N}(X), Y\right) \\
-c g\left(A_{F Y}(X), t N\right) .
\end{gathered}
$$

With the help of (2.2), (3.2), and (3.3), we derive

$$
\begin{gathered}
g\left(\nabla^{2} A(X), Y\right)=-g(A \circ \tilde{A}(X)+\underset{\sim}{A} \circ A(X), Y) \\
+\frac{c}{4}\left(n-3+14\left(n_{1} \cos ^{2} \theta_{1}+n_{2} \cos ^{2} \theta_{2}\right)\right) g\left(A_{N}(X), Y\right) \\
-c g\left(A_{F Y}(X), t N\right) .
\end{gathered}
$$


We calculate the preceding equation (3.4) for $X=e_{i}$ and $Y=A_{N}\left(e_{i}\right)$, we get

$$
\begin{gathered}
g\left(\nabla^{2} A\left(e_{i}\right), A_{N}\left(e_{i}\right)\right)=-g\left(A \circ \tilde{A}\left(e_{i}\right)+\underset{\sim}{A} \circ A\left(e_{i}\right), A_{N}\left(e_{i}\right)\right) \\
+\frac{c}{4}\left(n-3+14\left(n_{1} \cos ^{2} \theta_{1}+n_{2} \cos ^{2} \theta_{2}\right)\right) g\left(A_{N}\left(e_{i}\right), A_{N}\left(e_{i}\right)\right) \\
-c g\left(A_{F A_{N}\left(e_{i}\right)}\left(e_{i}\right), t N\right) .
\end{gathered}
$$

Again applying Lemmas 3.1-3.3 and by summation for $i=1, \ldots, n$, we find

$$
\sum_{i=1}^{n} g\left(A_{F A_{N}\left(e_{i}\right)}\left(e_{i}\right), t N\right)=-\left(1-2\left(n_{1} \cos ^{2} \theta_{1}+n_{2} \cos ^{2} \theta_{2}\right)\right)\|A\|^{2} .
$$

Thus, our assertion (3.1) follows on combining equations (3.5) and (3.6).

The following is Simons' type formula for a hemi-slant submanifold in $\bar{M}(c)$.

Corollary 3.5. Let $M$ be an $n$-dimensional minimal hemi-slant submanifold in a complex space form $\bar{M}(c)$ of dimension $2 n$. If $\bar{\nabla} P=0$, then

$$
g\left(\nabla^{2} A, A\right)=-g(A \circ \tilde{A}+\underset{\sim}{A} \circ A, A)+\frac{c}{4}\left[n+1+6 n_{2} \cos ^{2} \theta\right]\|A\|^{2} .
$$

For a semi-slant submanifold in $\bar{M}(c)$, we have the following Simons' type formula.

Corollary 3.6. Let $M$ be an n-dimensional minimal semi-slant submanifold in a complex space form $\bar{M}(c)$ of dimension $2 n$. If $\bar{\nabla} P=0$, then

$$
g\left(\nabla^{2} A, A\right)=-g(A \circ \tilde{A}+\underset{\sim}{A} \circ A, A)+\frac{c}{4}\left[n+1+6\left(n_{1}+n_{2} \cos ^{2} \theta\right)\right]\|A\|^{2} .
$$

\section{Main results}

This section gives the applications of Simons' type formula which has been derived in the last section for different submanifolds in the same ambient space. At this moment, we need to construct an inequality for minimal bi-slant submanifold in $\bar{M}(c)$, which shall be used in proving our main theorem.

Lemma 4.1. Let $M$ be an $n$-dimensional minimal bi-slant submanifold of a complex space form $\bar{M}(c)$ of dimension $2 n$ such that $c>0$. If $\bar{\nabla} P=0$, then

$$
g\left(\nabla^{2} A, A\right) \geq\left\{\left(-2+\frac{1}{n}\right)\|A\|^{2}+\frac{c}{4}\left[n+1+6\left(n_{1} \cos ^{2} \theta_{1}+n_{2} \cos ^{2} \theta_{2}\right)\right]\right\}\|A\|^{2} .
$$

Proof. Using Lemma 5.3.1 of J. Simons ([19], pg - 93), i.e.,

$$
g(A \circ \tilde{A}+\underset{\sim}{A} \circ A, A) \leq\left(2-\frac{1}{n}\right)\|A\|^{4}
$$

in Theorem 3.4, we find

$$
g\left(\nabla^{2} A, A\right) \geq\left(-2+\frac{1}{n}\right)\|A\|^{4}+\frac{c}{4}\left[n+1+6\left(n_{1} \cos ^{2} \theta_{1}+n_{2} \cos ^{2} \theta_{2}\right)\right]\|A\|^{2} .
$$

Thus, our assertion follows.

At this stage, we are ready to prove our main result by using the developed inequality (4.1) and well known Hopf's Lemma. 
Theorem 4.2. Let $M$ be an $n$-dimensional compact minimal bi-slant submanifold of a complex space form $\bar{M}(c)$ of dimension $2 n$ such that $c>0$ and $\bar{\nabla} P=0$. If the second fundamental form A satisfies

$$
\|A\|^{2} \leq \frac{c}{4\left(2-\frac{1}{n}\right)}\left(n+1+6\left(n_{1} \cos ^{2} \theta_{1}+n_{2} \cos ^{2} \theta_{2}\right)\right),
$$

then either $M$ is totally geodesic or

$$
\|A\|^{2}=\frac{c}{4\left(2-\frac{1}{n}\right)}\left(n+1+6\left(n_{1} \cos ^{2} \theta_{1}+n_{2} \cos ^{2} \theta_{2}\right)\right) .
$$

Proof. Since $M$ is compact, we have

$$
\int_{M} g\left(\nabla^{2} A, A\right)=-\int_{M} g(\nabla A, \nabla A) .
$$

Using Lemma 4.1 and (4.2), it follows that

$$
\begin{gathered}
0 \leq \int_{M} g(\nabla A, \nabla A) \\
\leq \int_{M}\left\{\left[\left(2-\frac{1}{n}\right)\|A\|^{2}-\frac{c}{4}\left(n+1+6\left(n_{1} \cos ^{2} \theta_{1}+n_{2} \cos ^{2} \theta_{2}\right)\right)\right]\|A\|^{2}\right\} .
\end{gathered}
$$

So, (4.3) is known as an integral formula of Simons' type. Further, by considering the hypothesis of the theorem, we find $\nabla A=0$ and hence $\nabla\|A\|^{2}$ is a constant, i.e., either $\|A\|^{2}=0$ or

$$
\|A\|^{2}=\frac{c}{4\left(2-\frac{1}{n}\right)}\left[n+1+6\left(n_{1} \cos ^{2} \theta_{1}+n_{2} \cos ^{2} \theta_{2}\right)\right] .
$$

From $A=0$, we conclude that $M$ is totally geodesic. Hence, our assertions follow.

Since we know that bi-slant submanifolds generalize the hemi-slant and semi-slant submanifolds, therefore, we can easily state the following theorem by using Corollary 3.5.

Theorem 4.3. Let $M$ be an n-dimensional compact minimal hemi-slant submanifold of a complex space form $\bar{M}(c)$ of dimension $2 n$ such that $c>0$ and $\bar{\nabla} P=0$. If the second fundamental form A satisfies

$$
\|A\|^{2} \leq \frac{c}{4\left(2-\frac{1}{n}\right)}\left[n+1+6 n_{2} \cos ^{2} \theta\right],
$$

then either $M$ is totally geodesic or

$$
\|A\|^{2}=\frac{c}{4\left(2-\frac{1}{n}\right)}\left[n+1+6 n_{2} \cos ^{2} \theta\right] .
$$

The following theorem can be stated by using Corollary 3.6.

Theorem 4.4. Let $M$ be an $n$-dimensional compact minimal semi-slant submanifold of a complex space form $\bar{M}(c)$ of dimension $2 n$ such that $c>0$ and $\bar{\nabla} P=0$. If the second fundamental form A satisfies

$$
\|A\|^{2} \leq \frac{c}{4\left(2-\frac{1}{n}\right)}\left(n+1+6\left(n_{1}+n_{2} \cos ^{2} \theta\right)\right),
$$

then either $M$ is totally geodesic or

$$
\|A\|^{2}=\frac{c}{4\left(2-\frac{1}{n}\right)}\left(n+1+6\left(n_{1}+n_{2} \cos ^{2} \theta\right)\right) .
$$

As the ambient manifold is a complex space form $\bar{M}(c)$, we can take a complex projective space $C P^{2 n}$ with constant holomorphic sectional curvature 4 of real dimension $2 n$. Then the following corollary follows from Theorem 4.2 and Lemma 4 of K. Abe [1]. 
Corollary 4.5. Let $M$ be an $n$-dimensional complete, compact minimal bi-slant submanifold of $C P^{2 n}$. If the second fundamental form A satisfies

$$
\|A\|^{2}<\left(\frac{n+1+6\left(n_{1} \cos ^{2} \theta_{1}+n_{2} \cos ^{2} \theta_{2}\right)}{2-\frac{1}{n}}\right),
$$

then $M$ is either a

(1) complex projective space, or

(2) real projective space.

In the same manner, we can have the following corollaries based on Theorems 4.3 and 4.4 , respectively.

Corollary 4.6. Let $M$ be an n-dimensional complete, compact minimal hemi-slant submanifold of $C P^{2 n}$. If the second fundamental form A satisfies

then $M$ is either a

$$
\|A\|^{2}<\left(\frac{n+1+6 n_{2} \cos ^{2} \theta}{2-\frac{1}{n}}\right),
$$

(1) complex projective space, or

(2) real projective space.

Corollary 4.7. Let $M$ be an n-dimensional complete, compact minimal semi-slant submanifold of $C P^{2 n}$. If the second fundamental form A satisfies

$$
\|A\|^{2}<\left(\frac{n+1+6\left(n_{1}+n_{2} \cos ^{2} \theta\right)}{2-\frac{1}{n}}\right),
$$

then $M$ is either a

(1) complex projective space, or

(2) real projective space.

Acknowledgment. The authors thank the referees for many valuable suggestions to improve the presentation of this article.

\section{References}

[1] K. Abe, Applications of Riccati type differential equation to Riemannian manifolds with totally geodesic distributions, Tohoku Math. J. 25 (2), 425-444, 1973.

[2] A. Bejancu, Geometry of CR-submanifolds, Mathematics and its Applications, (East European Series), 23. D. Reidel Publishing Co., Dordrecht, 1986.

[3] A. Bejancu, M. Kon and K. Yano, CR-submanifolds of a complex space form, J. Differential Geom. 16 (1) 137-145, 1981.

[4] J.L. Cabrerizo, A. Carriazo, L.M. Fernández and M. Fernández, Semi-slant submanifolds of a Sasakian manifold, Geom. Dedicata 78 (2), 183-199, 1999.

[5] J.L. Cabrerizo, A. Carriazo, L.M. Fernández and M. Fernández, Slant submanifolds in Sasakian manifolds, Glasg. Math. J. 42 (1), 125-138, 2000.

[6] J.L. Cabrerizo, A. Carriazo, L.M. Fernández and M. Fernández, Structure on slant submanifold of a contact manifold, Indian J. Pure Appl. Math. 31 (7), 857-864, 2000.

[7] A. Carriazo, Bi-slant immersions, in: Proc. ICRAMS, 88-97, Kharagpur, India, 2000.

[8] B.-Y. Chen, Geometry of Submanifolds, Pure and Applied Mathematics, No. 22. Marcel Dekker, Inc., New York, 1973.

[9] B.-Y. Chen, Geometry of slant submanifolds, Katholieke Universiteit Leuven, Louvain, 1990.

[10] B.-Y. Chen, Slant immersions, Bull. Aust. Math. Soc. 41 (1), 135-147, 1990. 
[11] B.-Y. Chen and D.E. Blair, On CR submanifolds of Hermitian manifolds, Israel J. Math. 34 (4), 353-363, 1979.

[12] B.-Y. Chen and S. Uddin, Warped product pointwise bi-slant submanifolds of Kaehler manifolds, Publ. Math. Debrecen 92, (1-2), 183-199, 2018.

[13] M. Gülbahar, E. Kılıç, and S. Keleş, A useful orthonormal basis on bi-slant submanifolds of almost Hermitian manifolds, Tamkang J. Math. 47 (2), 143-161, 2016.

[14] T. Ikawa, On submanifolds in a space with Sasakian 3-structure, Colloq. Math. 47 (1), 47-57, 1982.

[15] T. Ikawa, On some C-totally real submanifolds in a space with Sasakian 3-structure, Colloq. Math. 49 (1), 27-32, 1984.

[16] A. Mihai and I. Mihai, CR-submanifolds in complex and Sasakian space forms, in: Geometry of Cauchy-Riemann Submanifolds, 217-266, Springer, Singapore, 2016.

[17] N. Papaghiuc, Semi-slant submanifolds of a Kaehlerian manifold, An. Ştiint Al. I. Cuza Iaşi. Mat. 40 (1), 55-61, 1994.

[18] A.N. Siddiqui, M.H. Shahid and M. Jamali, Simons' type formula for Kaehlerian slant submanifolds in complex space forms, Kyungpook Math. J. 58 (1), 149-165, 2018.

[19] J. Simons, Minimal varieties in Riemannian manifolds, Ann. Math. 88 (2), 62-105, 1968.

[20] B. Şahin, Warped product submanifolds of Kaehler manifolds with a slant factor, Ann. Polon. Math. 95 (3), 207-226, 2009.

[21] S. Yamaguchi, M. Kon and T. Ikawa, C-totally real submanifolds, J. Differential Geometry, 11 (1), 59-64, 1976.

[22] K. Yano and M. Kon, CR submanifolds of Kaehlerian and Sasakian manifolds, Progress in Mathematics, 30. Birkhäuser, Boston, Mass., 1983.

[23] K. Yano and M. Kon, Structures on manifolds, Series in Pure Mathematics, 3. World Scientific Publishing Co., Singapore, 1984. 